\title{
Uso de las APPs con la tableta en la educación primaria
} y competencias asociadas

\section{Use of APPs on tablet in primary education and associated competences}

\author{
Cristina Sánchez -Martínez, María-Carmen Ricoy \\ Universidad de Vigo
}

\begin{abstract}
Resumen
A pesar de disponer actualmente de una gran cantidad de aplicaciones educativas (APPs), las investigaciones disponibles son escasas. Por ello, en este estudio se analiza su uso y competencias asociadas con la tableta en la Educación Primaria.

El trabajo se enmarca en la investigación cualitativa con la recogida de información a través del rastreo y seguimiento de un importante elenco de aplicaciones digitales. Como conclusiones cabe destacar que las asignaturas en las que se usan APP son Lengua y Literatura, Ciencias Sociales y Naturales. La competencia necesaria y, al mismo tiempo, la más desarrollada en el alumnado es la digital.

Palabras clave: APPs, competencia digital, educación primaria, contenidos curriculares.
\end{abstract}

\begin{abstract}
In spite of having a large range of educational applications at present, available studies are already limited. So this research analyses their use and the competences associated with tablet in Primary Education. The present work is part of the qualitative research with the data collected by means of the tracking and monitoring of a significant number of digital applications. In conclusion, those subjects which use APPs are Spanish Language and Literature, Social Sciences and Natural Sciences. The necessary and most developed competence among students is the digital competence.

Keywords: APPs, digital competence, Primary Education, curricular contents.
\end{abstract}

Con el desarrollo de Internet la tecnología se ha convertido en la protagonista de las sociedades avanzadas presentando un panorama en el que se cruza el plano de lo educativo y lo tecnológico. En la sociedad actual, la tecnología de pantalla táctil está tomando, cada vez, un papel más importante en la vida familiar, social, escolar y laboral. De hecho no sólo aumenta el número de dispositivos, en su uso general, sino que también lo hace con los niños-as y a edades, cada vez, más tempranas. En este sentido, es de indicar que el $75 \%$ de los niños estadounidenses, menores de ocho años, usan estos dispositivos y esa cantidad continuará aumentando (Rideout, 2013). De forma similar, ocurre en otros países occidentales (Mediawijzer, 2014). Debido a las altas tasas de acceso y uso de los dispositivos tecnológicos y de internet, no es de extrañar que las aplicaciones educativas y lúdicas se dirijan a un público infanto-juvenil y eleven su crecimiento, con más de cien mil aplicaciones disponibles (Godfrey y Reed, 2013). Sin embargo, hasta la fecha se sabe poco acerca de cómo los padres-madres y maestros-as deben seleccionar y usar las aplicaciones educativas digitales (Broekman, Piotrowskj, Beenties y Valkenburgm, 2016).

Siguiendo a Salmerón, Rodríguez y Gutiérrez (2010), nos encontramos ante un nuevo paradigma que relaciona las principales teorías del aprendizaje con los recursos tecnológicos. Estos autores enfatizan que los procesos de aprendizaje poseen un carácter eminentemente social, considerando que las Tecnología de la Información y Comunicación (TIC) son herramientas enriquecedoras para crear contextos interpersonales de aprendizaje. La influencia de las nuevas tecnologías se enfatiza en las generaciones más jóvenes surgiendo nuevos perfiles de consumidores. Prensky (2004) acuña los términos inmigrantes digitales y nativos digitales asociándolos con consumidores de edad infantil. El primero hace referencia a las personas que se han adaptado a la tecnología. Los segundos se identifican con aquellas personas que han crecido y se han desarrollado inmersos en la era digital. Estos nuevos usuarios entienden la tecnología como parte de sus vidas.

Recientemente se ha instalado en nuestro contexto un nuevo dispositivo como la tableta digital, que junto con el smartphone, comienza a acaparar el tiempo dedicado a ver la televisión o a utilizar los ordenadores portátiles, en lo que a consumo de contenidos se refieren. Sorprendentemente, el $10 \%$ de los niños y niñas menores de un año ya utilizan las pantallas inteligentes, el porcentaje se eleva al $39 \%$ en el caso de los menores de cuatro años y supera el 50\% en los mayores de ocho años (Sánchez-Labella, 2016). Además, en ese estudio se sostiene que de los más pequeños utilizan este dispositivo en torno al $77 \%$ para jugar, el $57 \%$ lo hace con propósitos educativos, el $55 \%$ para entretenerse durante los viajes, el $33 \%$ para ver películas y programas de televisión, el $41 \%$ para divertirse en restaurantes o eventos y el $15 \%$ para comunicarse con los amigos y la familia. Cabe destacar que el $46 \%$ de las aplicaciones descargadas son juegos que promueven únicamente la diversión, el $42 \%$ son juegos educativos, el 28\% promueven la creatividad de los niños y el $19 \%$ son apps basadas en personajes televisivos. 
Siguiendo a Gutiérrez-Cabello, Losada y Correa (2015) para que la inclusión de las TIC en el ámbito educativo sea exitosa es imprescindible el desarrollo de la competencia digital. Cabe destacar que para ello, es fundamental que el profesorado cuente con la formación necesaria para poder trabajar dicha competencia en el aula, así como con los medios y recursos suficientes. Tal y como afirman Littlejohn y Margaryan (2010) es inexcusable la preparación del alumnado, para que en un futuro sean ciudadanos competentes en la era digital.

\section{Diseño y metodología de investigación}

El objetivo general de este estudio es analizar el uso de las Aplicaciones educativas con la tableta digital en el currículo de Educación Primaria, así como las competencias asociadas a su utilización. Como objetivos específicos se pretende conocer las materias en las que predomina el uso de las Apps y descubrir las competencias asociadas al uso de las Apps con la tableta.

El trabajo abordado forma parte de una investigación más amplia y se enmarca en la metodología cualitativa (enfoque etnográfico-narrativo). Este tipo de investigación contribuye al desarrollo de su comprensión y genera nuevo conocimiento, partiendo de la observación sistemática, análisis de documentos, situación y hechos Dewalt y Dewalt (2002) consideran que la técnica de observación y el análisis de documentos ayudan, de forma objetiva y precisa, a una comprensión holística de la situación objeto de estudio. Esta modalidad permite la recogida de datos de modo estructurado, sin que necesariamente exista intrusismo en el contexto objeto de estudio (Taylor y Bogdan, 2010); así mismo Flick (2004) afirma que este tipo de investigación profundiza, desde diferentes vertientes en las particularidades del contexto sin limitarse a la mera descripción.

\section{Instrumentos, procedimiento y muestra}

Para desarrollar este estudio se rastreó en internet, durante cuatro meses, las 60 aplicaciones educativas (tabla 1) más descargadas y utilizadas en la actualidad en España, para la Educación Primaria. Para ello, se tuvieron en cuenta las dos plataformas online de las aplicaciones más importantes (App Store de Apple y Android). Las Apps estaban comentadas y valoradas críticamente, por un mínimo de dos comentarios y un máximo de treinta (con una media de cinco personas, tratándose de profesorado, padres o madres) de forma anónima, después de haberlas utilizado en alguna ocasión con los niños-as.

La muestra de análisis se ha realizado con las siguientes: Duolingo; Palabra correcta; Planeta Geo; Quiver: Tus dibujos ...; Slice it! ...; Cuadernos Rubio; Stellarium; Quix Tabla; ecoRecibla; Iberia: Ríos y Montes; CreAPPCuentos; El increíble chapuzón de Zoe; Diccionario desconectado; Lectura rápida; Horario de clase; Mozart Interactive; Bicis y cascos; Sky Map; Sopa de Letras Primaria; Disney Action!; Kids Doodle; TrashChaos Free; Matemáticas Link Up; Spanish Verbs Lite; Obras maestras de la música clásica; Atrapa un millón; Memorado: Juego cerebrales; La Biblia para niños; ArbolApp; Oído perfecto; Cabritos de
Matemáticas ...; Needit Feedit; GeoExpert HD Lite; Mounstruario; Catapum; Sopa de Letras Infinita; FotoFlo; El Guardián de la Imaginación HD; Palabras Domino Free; The Opposites; Europe Map Puzzle; Matemáticas Rápidas; Aprender Inglés ejercicios, vocabulario HD; Soy pintor; Europa Puzzle; Geo Trainer HD; Aprender Inglés: Memoriza Palabras; Aprender Inglés con Gymglish, ....; Inglés Verbos HD; Maps Jigsaw Puzzles; Entrenamiento del ritmo; Cubo Mágico 3D; Santi y Boini; Basic Fraction; Shop \& Math; Suma+; Piano Invention ...; Chicken Coop ...; Ana Lomba ...; Aprende Inglés con ABA English.

\section{Resultados}

A continuación se presentan los principales resultados extraídos del estudio.

\section{Uso de las Apps en las materias del currículum de} educación primaria

Continuamente emergen nuevas aplicaciones educativas, lo que hace que el abanico vaya siendo más amplio en la propia praxis escolar. Con todo, debido a la reciente incorporación de las tabletas en el contexto del aula y al incipiente uso de las Apps todavía no existen clasificaciones sólidas al respecto para aglutinarlas. Sin embargo, es interesante agrupar las distintas aplicaciones educativas atendiendo a alguna característica, componentes, contenido trabajado, formato, funciones, etc. De este estudio se desprende una clasificación de las Apps atendiendo al soporte y naturaleza de su contenido en las materias del currículum de Educación Primaria:

\section{Apps multimedia con contenidos propiamente curriculares}

- Tipo libro, enciclopedia y organizadores (ejemplo: Cuadernos Rubio; Horario de clase; Lectura rápida; Palabra correcta; Iberia: Ríos y Montes; GeoExpert HD Lite; Needit Feedit).

Diccionarios (ejemplo: Duolingo; Diccionario desconectado; Spanish Verbs Lite; Aprender Inglés: Memoriza Palabras; Inglés Verbos HD; Aprender Inglés: ejercicios, vocabulario HD).

\section{Apps multimedia con contenidos lúdicos asociados al currículum}

Tipo juegos (ejemplo: Quix Tabla; Slice it! Fracciones y geometría; Memorado: Juegos cerebrales; Atrapa un millón; Matemáticas Link Up; Sopa de Letras Primaria Disney Action!; Cabritos de Matemáticas Maniaco; Planeta Geo; Palabras Domino Free - juego de letras para los niños y los grandes; The Opposites; Europa Puzzle; Sopa de Letras Infinita; Chicken Coop Fractions Game; Basic Fraction; Suma+; Matemáticas Rápidas; Puzzles; Aprende Geografía; Geo Trainer HD; Santi y Boini).

- Para talleres lúdico-creativos (ejemplo: Quiver: Tus dibujos cobran vida; EcoRecibla Stellarium; Oído perfecto; ArbolApp; Obras maestras de la música clásica; TrashChaos Free; Kids Doodle; Sky Map; Bicis y cascos; Mozart Interactive; El Guardián de la Imaginación HD; Soy pintor; Europe-Puzzle; Aprender Inglés con Gymglish, la gimnasia del Inglés; Entrenamiento del 
ritmo; Shop \& Math; Maps Jigsaw Puzzles; Puzzles 3D; FotoFlo).

- Con cuentos (ejemplo: El increíble chapuzón de Zoe; CreAPPCuentos; La Biblia para niños; Catapum; Mounstruario; Ana Lomba - Inglés para niños: La gallina roja -Cuento bilingüe español-inglés-).

Del análisis realizado se desprenden ciertos patrones de uso de las aplicaciones digitales, en función del curso y las materias del currículum en las que se registra su utilización (figura 1). En los cursos iniciales ( $1^{\circ}$ y $2^{\circ}$ curso) de Educación Primaria es en los que está más instaurada la inclusión de Apps. A su vez, en éstos se utilizan de forma más extensible incluyéndolas, aunque de modo desigual, en las diferentes materias del currículum escolar: Ciencias Naturales y CC. Sociales; Educación Artística; Educación Física, Leguas y Literaturas; Matemáticas y Religión/Educación en Valores.

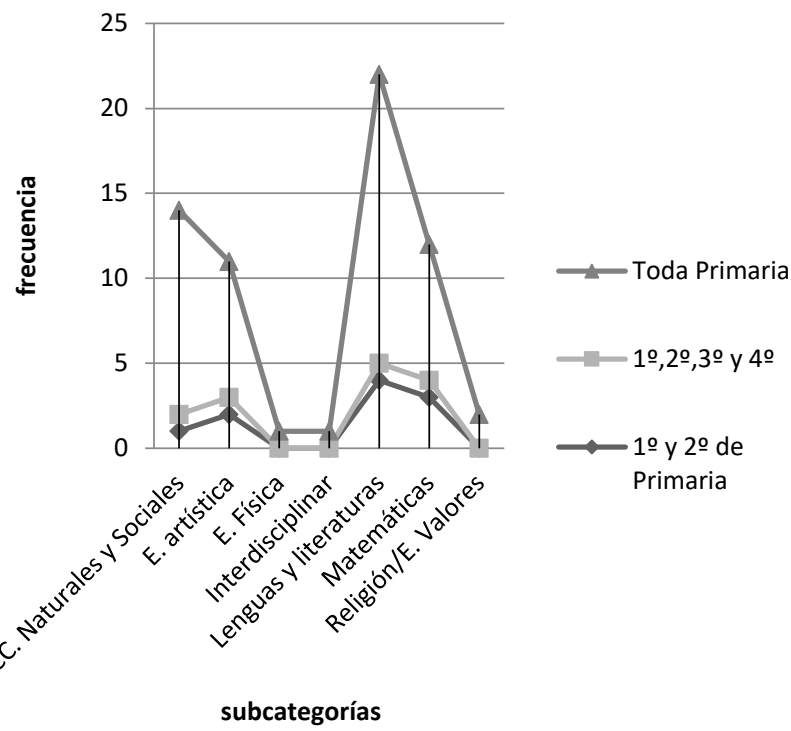

Figura 1. Uso de las Apps en las materias del currículum

En las materias de Lenguas y Literaturas (tanto del idioma español, como del inglés y gallego) es en las que existe una mayor tendencia de la utilización de las Apps. Este hecho puede ser provocado por el reciente crecimiento de la oferta de aplicaciones educativas (Duolingo, Spanish Verbs Lite, Aprender Inglés con Gymglish, la gimnasia del Inglés ...) asociadas con el aprendizaje de una nueva lengua, en particular en el caso de España, para la lengua inglesa.

Las actividades que se realizan con una mayor notoriedad con las Apps se focalizan a la traducción de frases del idioma extranjero, la elaboración de redacciones cortas y la escucha de conversaciones sencillas o cuentos. Además con la aplicación de Palabra Correcta se le plantea que, completen frases incompletas con el objeto de aumentar el vocabulario. En este sentido, una de las ventajas que encuentra el profesorado en estas Apps es que facilitan el aprendizaje personalizado al alumnado, permitiendo de este modo que se respeta su nivel académico y ritmo de trabajo. Con todo, el tipo de praxis no difiere demasiado de la asociada a la metodología tradicional.

A través de las Apps (ecoRecibla, Stellarium, Iberia: Ríos y Montes, ArbolApp) el alumnado de Educación Primaria desarrolla diferentes actividades en la materia de Ciencias Naturales y en la de CC. Sociales. Las Apps utilizadas le permiten trabajar primordialmente contenidos de tipo procedimental y actitudinal asociados con el desarrollo de hábitos, el disfrute y cuidado del medio ambiente. En este sentido las prácticas están dirigidas principalmente a reforzar su ubicación espacial, promover la conciencia crítica y la generación de actitudes cívicas, a partir del ahorro energético y el uso apropiado de los recursos naturales. A modo de ejemplo puede indicarse la realización de actividades sobre el: reciclado de residuos en el contenedor adecuado (azul, verde y amarillo); observaciones en $3 \mathrm{D}$ para generar debates sobre el deterioro del planeta; localización geográfica de las comunidades autónomas españolas, de capitales y provincias, ríos y otros componentes espaciales.

Entre las materias del currículum escolar en las que se realiza un uso excepcional de las Apps se encuentran la de Educación Física, así como el trabajo planteado desde el abordaje de contenidos Interdisciplinares. Solamente una de las aplicaciones analizadas (Bicis y Cascos) se vincula con la materia de Educación Física. Las actividades de esta App permiten al alumnado desarrollar contenidos y competencias sobre Educación Vial, a través del uso de recursos multimedia con la presentación de simulaciones sobre la circulación en bicicleta de forma segura. Con las Apps denominadas "Toca la señal", "rompecabici" y "un paseo seguro en bici" se trabajan aspectos para diferenciar las distintas señales de tráfico, la identificación de las partes de una bicicleta, así mismo posibilitan la realización de la autoevaluación sobre los conocimientos viales trabajados.

\section{Competencias asociadas al uso de las Apps con la tableta}

La LOMCE (2013) establece para el alumnado de Educación Primaria las siguientes competencias clave:

- Comunicación lingüística;

- Competencia matemática y competencias básicas en ciencia y tecnología;

- Competencia digital;

- Aprender a aprender;

- Competencias sociales y cívicas;

- Sentido de iniciativa y espíritu emprendedor;

- Conciencia y expresiones culturales

La referida clasificación es la que se ha tenido en cuenta en el análisis para la sistematización de los datos posibilitados por los participantes sobre el uso de las Apps. Ello, permite delimitar las competencias que, desde la percepción de los expertos, le resultan necesarias al alumnado, como punto de partida, para utilizar las Apps en la tableta y aquellas que desarrollan con su uso (tabla 2).

Para desarrollar la competencia digital, tal como se observa en la tabla 2, son primordiales las competencias de aprender a aprender (que posibilita fundamentalmente una mayor autonomía y sentido crítico). La competencia 
de Aprender a Aprender sensatamente se revela de forma acentuada. Su importancia es esencial para promover la capacidad para iniciar, organizar y persistir en el aprendizaje, aspectos también imprescindibles para desarrollar la competencia digital, debido a las transformaciones o cambios que, hoy más que nunca, caracterizan a las nuevas tecnologías.

Por otra parte, se produce una confluencia entre la competencia digital y la competencia de concienciación y expresiones culturales (que favorece principalmente el desarrollo del sentido crítico y la apertura a la

Tabla 2.

Confluencia entre las competencias iniciales y las desarrolladas con las Apps multiculturalidad, el respeto a las demás personas y la capacidad para disfrutar del patrimonio cultural). Así mismo, es necesario para desarrollar el espíritu crítico una actitud abierta ante/con las TIC, considerándolas también parte de nuestra riqueza cultural. La competencia matemática y las competencias en ciencia y tecnología requieren del fomento de destrezas digitales e incluyen actitudes y valores relacionados con la asunción de criterios éticos, relativos a la ciencia y a la tecnología.

\begin{tabular}{|c|c|c|c|c|c|c|c|c|}
\hline TABLETA Y APPS & 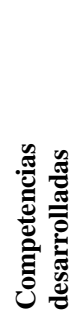 & 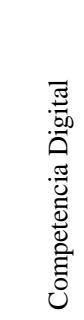 & 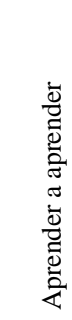 & 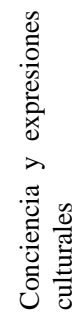 & 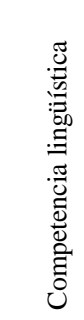 & 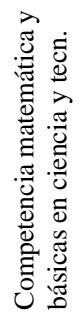 & 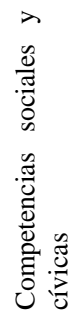 & 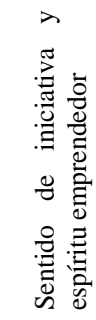 \\
\hline \multicolumn{9}{|l|}{ Competencias necesarias } \\
\hline Competencia Digital & & 60 & 12 & 44 & 28 & 40 & 36 & 4 \\
\hline Aprender a Aprender & & 58 & 12 & 44 & 24 & 40 & 36 & 4 \\
\hline Conciencia y expresiones culturales & & 43 & 12 & 48 & 20 & 28 & 28 & \\
\hline Competencia lingüística & & 22 & 12 & 8 & 28 & 8 & 4 & \\
\hline Compet. matemática y en ciencia y tecnología & & 36 & 4 & 8 & & 20 & 4 & 4 \\
\hline Competencias sociales y cívicas & & 36 & 4 & 16 & & 4 & 36 & \\
\hline Sentido de iniciativa y espíritu emprendedor & & 24 & & & 4 & 4 & 4 & 4 \\
\hline
\end{tabular}

Leyenda: En las columnas se representan las competencias desarrolladas con la tableta con las Apps y en las filas las competencias necesarias, como punto de partida, que le resultan imprescindibles alumnado para continuar progresando. $\mathrm{Su}$ frecuencia es el resultado de la confluencia entre la/a competencia/s necesaria/s, como punto de partida, y la/s competencia/s desarrollada/s en el proceso de aprendizaje con la tableta y las Apps.

Cabe señalar que las competencias sociales y cívicas exigen habilidades y capacidades múltiples para convivir con los demás, comprender, interpretar y resolver los problemas en la sociedad digital. Por otro lado, es de señalar que la competencia digital también resulta necesaria para desarrollar la competencia de concienciación y expresión cultural, la competencia en matemáticas y en ciencia y tecnología, así como la competencia social y cívica. Finalmente, hay que resaltar que además de lo referido para avanzar competencialmente es necesario que el alumnado desarrolle una actitud positiva general sobre los avances tecnológicos a lo largo del tiempo.

\section{Discusiones y Conclusiones}

De forma global, cabe indicar que en el contexto objeto de estudio el impacto positivo de la tableta y las Apps, en el proceso de aprendizaje del alumnado de Educación Primaria, es todavía escaso. Su inclusión no se encuentra normalizada en la implementación curricular, aunque figura sutilmente en los diseños didácticos del profesorado. La tableta y las Apps todavía no han desbancado el uso del libro de texto y los docentes únicamente se plantean su integración, de forma excepcional, y como mero apoyo o complemento del recurso hegemónico.

Cabe resaltar que el alumnado a través de la tableta puede acceder a una mayor cantidad de información actualizada; aunque la existencia de un incremento en la flexibilidad del proceso de enseñanza-aprendizaje no se pone de manifiesto en este estudio. Es de señalar, según Cánovas (2014), que desde la escuela se tendría que promover una educación basada en la realidad, la creatividad, la generación de contenidos e ideas que contribuyan a transformar la educación y la sociedad en la que crecen los niños, con el apoyo de las TIC.

Ante la inexistencia de una clasificación sólida sobre las Apps para el aprendizaje, de este estudio se desprende una categorización precedente, en base a la organización de las distintas aplicaciones educativas, que se utilizan con la tableta con el alumnado de Educación Primaria. Desprendiéndose su agrupación del uso realizado en las materias del currículum de E. Primaria. Cabe resaltar que las Apps más utilizadas, en el contexto escolar, se asocian con la materia de Lenguas y Literaturas, la de Ciencias Sociales y la de CC. Naturales. Estos resultados pueden ser propiciados por el crecimiento de aplicaciones educativas que se utilizan para el aprendizaje de segundas lenguas, como idioma extranjero, especialmente en el caso de la Lengua Inglesa. En cuanto a las aplicaciones asociadas a la 
asignatura de Ciencias Naturales y a la de CC. Sociales, hay que señalar que predominan las aplicaciones acerca del cuidado del medio ambiente y de localización de capitales y provincias, así como sobre ríos y otros elementos geográficos del contexto español.

Hay que hacer hincapié en que aunque las tabletas crean nuevas oportunidades de aprendizaje, no pueden utilizase de cualquier manera si se pretende asegurar el desarrollo de competencias (Northrop, 2013). Asimismo, se debe ser consciente de que en ocasiones la tecnología puede distraer del objetivo principal (Chiong et. al., 2012). Con todo el desarrollo de competencias con la misma es, en sí mismo, una fuente de conocimiento valioso.

Del estudio realizado se desprende, con buena lógica, que entre las competencias iniciales necesarias y las desarrolladas para el uso de la tableta digital en Educación Primaria destaca, en ambos casos, la Competencia Digital. En el mismo sentido, es también la que los expertos consideran imprescindible y la que en la actualidad más acrecienta el alumnado. Hay que incidir en que esta competencia es fundamental para realizar un uso seguro y analítico de las TIC así como para obtener, producir e intercambiar información con otros usuarios. Con todo, para desarrollar la competencia digital es primordial también la competencia de Aprender a Aprender, Concienciación y Expresión cultural, la Competencia Matemática y en Ciencia y Tecnología y la Competencia Social y Cívica. Es interesante destacar cómo existe una retroalimentación entre las competencias necesarias, como punto de partida, y las desarrolladas y la repercusión de la Competencia Digital sobre las demás y a la inversa.

Las tabletas digitales son recursos que cuentan con un alto grado de interactividad por su pantalla táctil y sus posibilidades intuitivas, que apenas exigen una capacitación previa y se acoplan con facilidad con las capacidades que los usuarios hayan desarrollado con el uso de otros dispositivos móviles (Marés, 2012). Por este motivo la inclusión de estos dispositivos en el aula no puede pasar desapercibido. Además los educadores/maestros deben aprovechar esta herramienta y las aplicaciones educativas, que permiten trabajar contenidos en todas las asignaturas del currículum de un modo interactivo, creativo y divertido. A su vez, este dispositivo y las apps se presentan como un excelente medio para mejorar e incrementar la motivación del alumnado y facilitan el desarrollo de las competencias clave en el alumnado de Educación primaria.

\section{Agradecimientos}

Aprovechamos para manifestar el agradecimiento al Ministerio de Educación, Cultura y Deporte, por la financiación de esta investigación con el desarrollo de la Tesis Doctoral sobre el uso de las tabletas en la Educación Primaria.

\section{Referencias}

Broekman, F., Piotrowskj, J., Beenties, H. Y Balkenburg, P. (2016). A parental perspective on apps for young children. Computers in human behaviour, 63, 142-151.
Cánovas, G. (2014). Menores de edad y conectividad móvil en España: Tablets y smartphones. Accessed July $20 \quad 2013$. Http://www.diainternetsegura.es/descargas/estudio_m ovil_smartphones_tablets_v2c.pdf

Chiong, C., Ree, J., Takeuchi, L. Y Erickson, I. (2012). Print books vs. E-books: Comparing parent-child co-reading on print, basic, and enhanced e-book platforms. New York: The Joan Ganz Cooney Center

Dewalt, K. Y dewalt, B. (2002). Participant observation: a guide for fieldworkers. Walnut Creek, CA: altamira Press.

Godfrey, B. J. Y Reed, M. (2013). App store after five years. Recuperado de: http://actonline.org/wp-content/uploads/2014/04/TheApp-Store-After-Five-Years.pdf

Guitiérrez- Cabello, A, Losada, D. Y Correa, J. M. (2015). Concepciones previas de los estudiantes de Grado en Educación Primaria sobre la competencia digital de los escolares. Educatio Siglo XXI, 33(1), 235-257. Doi: http://dx.doi.org/10.6018/j/222581

Littlejohn, A. Y Margarayn, A. (2010). Sharing resources in educational communities. International Journal of Emerging Technologies in Learning, 5(2), 25-30. Doi:10.3991/ijet.v5i2.857

Lomce (2013). Ley Orgánica para la Mejora de la Calidad Educativa (Ley Orgánica 8/2013, 9 de diciembre). Boletín Oficial del Estado, nº 295 de 10 de diciembre.

Marés, L. (2012). Tablets en educación. Oportunidades y desafíos en políticas uno a uno. Relpe. OEI. Oficina Regional de Buenos Aires.

Mediawijzer (2014). Iene miene media: Een onderzoek naar mediagebruik door kleine kinderen [Research on media use by young children]. Recuperado de: http://www.mediawijzer.net/wp-content/uploads/iene miene_media_2014.pdf

Northrop, L. Y Killen, E. (2013). A framework for using ipads to build early literacy skills. The Reading Teacher, 66(7), 531-537

Prensky, M. (2004). The death of command and control? Montreal, Canadá: Technology Alliance Partners.

Rideout, V. (2013). Zero to eight: Children's media use in America 2013. Common Sense Media.

Salmerón, H., Rodríguez, S. Y Gutierrez, C. (2010). Metodologías que optimizan la comunicación en entornos de aprendizaje virtual. Comunicar, 34, 163-171

Sánchez-Labella, I. (2016). Las tablets como herramientas educativas para la infancia. Neox Kidz, un estudio de caso. Redes, 12, 47-67.

Taylor, S. J. Y Bogdan, R. (2010). Introducción a los métodos cualitativos de investigación: la búsqueda de significados. Barcelona: Paidós. 